\title{
ON THE CRITICAL TEMPERATURE OF SUPERCONDUCTORS
}

\author{
S. J. Poon
}

4975

JUNE 1975

\section{A REPORT ON RESEARCH CONDUCTED UNDER CONTRACT FOR THE \\ U.S. ATOMIC ENERGY COMMISSION}

FHERE IS NO OBJECTION FROM THE PATENT

STANDPOINT TO THE PUBLICATION OR

DISSEMINATION OF THIS DOCUMENT:

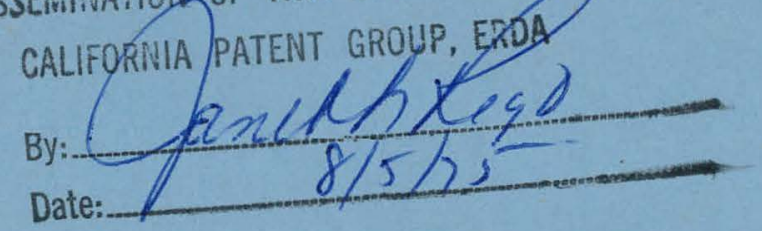

\section{W. M. KECK LABORATORY OF ENGINEERING MATERIALS}

CALIFORNIA INSTITUTE OF TECHNOLOGY

PASADENA 


\section{DISCLAIMER}

This report was prepared as an account of work sponsored by an agency of the United States Government. Neither the United States Government nor any agency Thereof, nor any of their employees, makes any warranty, express or implied, or assumes any legal liability or responsibility for the accuracy, completeness, or usefulness of any information, apparatus, product, or process disclosed, or represents that its use would not infringe privately owned rights. Reference herein to any specific commercial product, process, or service by trade name, trademark, manufacturer, or otherwise does not necessarily constitute or imply its endorsement, recommendation, or favoring by the United States Government or any agency thereof. The views and opinions of authors expressed herein do not necessarily state or reflect those of the United States Government or any agency thereof. 


\section{DISCLAIMER}

Portions of this document may be illegible in electronic image products. Images are produced from the best available original document. 
California Institute of Technology

W. M. Keck Laboratory of Engineering Materials

ON THE CRITICAL TEMPERATURE OF SUPERCONDUCTORS

by

S. J. Poon

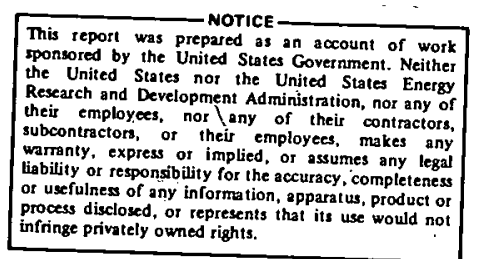

AEC Research and Development Report No. 65, under Contract No. AT(04-3)-822

Professor Pol Duwez, principal investigator. 


\section{NOTICE}

This report was prepared as an account of work spon-. sored by the United States Government. Neither the United States nor the United States Atomic Energy Commission, nor any of their employees, nor any of their contractors, subcontractors, or their employees, makes any warranty, express or implied, or assumes any legal liability or responsibility for the accuracy, completeness or usefulness of any information, apparatus, product or process disclosed, or represents that its use would not infringe privately-owned rights. 
An exact expression for the superconducting transition temperature is derived from the Eliashberg equation at $T=T_{c}$ taking the energy gap function in the two-square-well form. The expression is improved for self-consistency by using available tunneling data. It is found to give more restricted values of $T_{c}$ than the McMillan equation. The prospects of obtaining high $\mathrm{T}_{c}$ in alloy systems a re discussed within the framework of the new equation. 


\section{INTRODUCTION}

The fundamental theory of superconductivity of Bardeen, Cooper and Schrieffer has been well established. The trends of superconductivity in metals and alloys have been discovered and explained by several authors. $2,3,4$ It is possible to determine the transition temperature $T_{c}$ of superconductors with reasonable accuracy once the properties of the normal state of the solids are known. In fact, McMillan ${ }^{5}$ derived a formula for $T_{c}$ using the "strong-coupled" theory 6,7 as a function of materials parameters. However, as pointed out by McMillan, the formula should be treated with caution whenever there is no information available for the phonon density of states. Therefore, one should not take the predicted maximum $T_{c}$ in the paper too seriously since the coupling constant then exceeds unity. Instead, the idea of enhancing $T_{c}$ by increasing the coupling constant through a reduced mean square phonon frequency, or the so called "softening of phonon modes" is interesting. Since then, the problem of maximizing $T_{c}$ in solids has been discussed by other authors. Some proposals involved other mechanisms other than the electron-phonon interaction which are beyond the scope of the present discussion. Others related high $\mathrm{T}_{\mathrm{c}}$ to lattice instability. 8,9 However, the negative effect of low frequency phonons on $T_{c}$ was not pointed out until Allen. ${ }^{10}$ He found that phonons of frequency less than $T_{c}$ give a repulsive electron-phonon interaction, while phonons of frequency less than $4 \mathrm{~T}_{\mathrm{c}}$ have a diminished attraction. Recently, Johnson.. and Poon 11 used a phenomenological approach to study the trends of superconductivity in transition metal (TM) alloys. They found that $T_{c}^{\max }$ in TM alloys are determined by the melting temperature of the 
alloys in addition to the valence electrons per atom rules. It was concluded that $T_{C}$ in solids is probably limited to about $30{ }^{\circ}$. In this paper, we derive an exact expression for $T_{c}$ from a numerical solution of the Eliashberg equation at $T=T_{c}$ using a two-square-well energy gap function. Part of the derivation has been done by Allen. ${ }^{10}$ The expression is improved for self-consistency by fitting it to available tunneling data. The prospects of obtaining high $\mathrm{T}_{\mathrm{c}}$ in various alloy systems are discussed on the basis of our new equation.

In section II, we write the Eliashberg equation at $T=T_{c}$ for a general superconductor and give an exact solution using a particular gap model. The scheme is similar to Allen's. ${ }^{10}$ In section III, we rewrite our equation in a more convenient form by a curve fitting process. The equation is still rather general for the gap model we choose. In section IV, self-consistency of the equation is improved by fitting it to tunneling data of various elements and alloys. An equation which fits a wide variety of phonon spectra is obtained. It is regarded as our central $\mathrm{T}_{\mathrm{c}}$ equation. In the last section, the equation is used as a guide for investigating prospective alloy systems.

\section{THEORY}

In this section, we derive a formula for $\mathrm{T}_{c}$ following the scheme of Allen. 10 The notations used here will be the same as those in reference 5 and 10 unless otherwise specified. The derivation is based on the Eliashberg equation at $T=T_{c}$ which is accurate to order $(m / M)^{0.5}$. We also assume the pairing self-energy $\Delta(\omega)$ to be a two-square-well function of $\omega$. In mathematical form 


$$
\begin{aligned}
\Delta(\omega)= & Z^{-1}(\omega) \int_{0}^{\infty} \frac{d \omega^{\prime}}{\omega^{\prime}} \operatorname{Re}\left\{\Delta\left(\omega^{\prime}\right)\right\} \int_{0}^{\omega_{0}} d \omega_{q} \alpha^{2} F\left(\omega_{q}\right) \\
& \times\left\{\frac{N\left(\omega_{q}\right)+f\left(-\omega^{\prime}\right)}{\omega^{\prime}+\omega_{q}+\omega}+\frac{N\left(\omega_{q}\right)+f\left(-\omega^{\prime}\right)}{\omega^{\prime}+\omega_{q}-\omega}\right. \\
& \left.+\frac{N\left(\omega_{q}\right)+f\left(\omega^{\prime}\right)}{\omega^{\prime}-\omega_{q}+\omega}+\frac{N\left(\omega_{q}\right)+f\left(\omega^{\prime}\right)}{\omega^{\prime}-\omega_{q}-\omega}\right\} \\
& -Z^{-1}(\omega) \int_{0}^{E_{B}} \frac{d \omega^{\prime}}{\omega^{\prime}} \operatorname{Re}\left\{\Delta\left(\omega^{\prime}\right)\right\}\left[1-2 f\left(\omega^{\prime}\right)\right] \\
\Delta(\omega)= & \begin{cases}\Delta \Delta_{0} & 0<<\omega_{0} \\
\Delta \infty & \omega_{0}<\omega\end{cases}
\end{aligned}
$$

where $\mathrm{Z}, \mathrm{N}$, and $\mathrm{f}$ are the renormalization, Bose, and Fermi functions evaluated at $\mathrm{T}=\mathrm{T}_{\mathrm{c}}$ respectively, $\alpha^{2} \mathrm{~F}\left(\omega_{\mathrm{q}}\right)$ is the electron-phonon inter action function, $\mu$ is the Fermi surface averaged Coulomb interaction, $\omega_{0}$ is the phonon cut off frequency, and $R e$ means the real part. The value of $T_{c}$ is determined as the highest temperature at which a nontrivial solution exists in equation (1).

Equation (1) is solved by setting $\omega=0$ for $\Delta_{0}$ and $\omega=\infty$ for $\Delta_{\infty}$. The expression for $\Delta_{\infty}$ is the same as McMillan's

$$
\Delta_{\infty}=-\mu * \Delta_{0} \ln \left(1.134 \omega_{0} / T_{c}\right)
$$

with

$$
\mu^{*}=\mu / 1+\mu \ln \left(E_{B} / \omega_{0}\right)
$$

defined as the "Coulomb pseudopotential". The expression for $\Delta_{0}$ is divided into three parts according to the different phonon contribution terms plus a Coulomb term. The Coulomb term $\Delta^{3}(0)$ equals $\Delta_{\infty} / Z_{0}$ as shown by McMillan. ${ }^{5}$ The phonon terms $\Delta^{1}(0)$ and $\Delta^{2}(0)$ require 
modifications from a physical standpoint. Allen ${ }^{10}$ showed that the coupling of electrons to phonons of frequency $\omega_{q}$ less than $T_{c}$ causes $T_{c}$ to diminish, and phonons of frequency less than about $4 T_{c}$ have a diminished attraction. The electron-phonon interaction is a retarded interaction while the Coulomb repulsion between electrons is instantaneous. When a macroscopic electron density fluctuation of wavevector $\vec{q}\left(|\vec{q}|<<k_{F}\right)$ and frequency $w$ arises in a lattice, the ions can follow in phase with the electronic disturbance if $\omega$ is below the natural frequency $\omega_{q}$ of the phonons. Electrons with energy $E_{F}>>\omega$ (i.e. adiabatically responding to the ionic motions) are then effectively attracted by the electronic disturbance. If $\omega>\omega_{\mathrm{q}}$, the ionic vibrations are $180^{\circ}$ out of phase with the perturbation, and electrons are thus effectively repelled. For small $\omega_{q}$ (or small $\left.|\vec{q}|\right)$, the phase space for attractive interaction is quite limited. Besides, the long wavelength components cannot overcome the Coulomb repulsion term at small $\omega$ under lattice stability requirement. This is consistent with the fact that. umklapp scattering (i.e. large $|\vec{q}|$ ) is required for large electron-phonon couplings. 4,8 If the characteristic frequency $w$ of electronic disturbance at $T=T_{c}$ is of the order $T_{c}$, then the frequency componets are attractive only for $\omega_{q}>T_{c}$ while they are repulsive for $\omega_{q}<T_{c}$. Both repulsive and diminishing effects can be accounted for in the phonon term $\Delta^{l}(0)$ contributed from electronic interaction energy within the range $\omega<\omega_{0}$ comparable to that of the phonons. The inclusion of the Bose function $N\left(\omega_{q}\right)$ in the numerator and $\omega^{\prime} \neq 0$ in the phonon propagators when evaluating $\Delta^{l}(0)$ will give these effects. The formula for $\Delta^{1}(0)$ is given by 10 


$$
\Delta_{0}^{1}=\frac{\Delta_{0}}{Z_{0}}\left\{\lambda \ln \left(\frac{1.134 \omega_{0}}{T_{c}}\right)-2 \int_{0}^{\omega_{0}} \frac{d \omega_{q}}{\omega_{q}} \alpha^{2} F\left(\omega_{q}\right) f\left(\omega_{q}, \omega_{0}, T_{c}\right)\right\}
$$

$$
\text { with } \begin{aligned}
f\left(\omega_{q}, \omega_{0}, T_{c}\right) & =\ln \left(\frac{1.134 \omega_{0}}{T_{c}}\right)-\left[N\left(\omega_{q}\right)+\frac{1}{2}\right] \ln \left|\frac{\omega_{0}-\omega_{q}}{\omega_{0}+\omega_{q}}\right| \\
& -\frac{1}{2} \ln \left|\frac{\omega_{0}^{2}}{\omega_{0}^{2}-\omega_{q}^{2}}\right| \tanh \left(\frac{\omega_{0}}{2 T_{c}}\right)+\frac{1}{2} \int_{0}^{\omega_{0}} d \omega^{\prime} \ln \left|\frac{\omega^{\prime 2}}{\omega^{\prime 2}-\omega_{q}^{2}}\right| \frac{d}{d \omega^{\prime}} \tanh \left(\frac{\omega^{\prime}}{2 T_{c}}\right)^{(6)}
\end{aligned}
$$

The only divergent term in equation (6) as $\omega_{q} \rightarrow \omega_{0}$ is $\lim _{\omega \rightarrow \omega_{0}}$ $\left.N\left(\omega_{q}\right) \ln \left|\omega_{q}{ }^{-\omega_{0}}\right| \simeq e^{-\omega_{0} / T}{\underset{\omega}{\omega_{q} \rightarrow \omega_{0}}}_{\lim _{q}} \ln _{q_{0}}\right|_{\omega_{0}} \mid$ due to the discontinuity in the model chosen for $\Delta$. This singularity can be removed by assuming that the electron-phonon interaction function $\alpha^{2} F\left(\omega_{q}\right) \rightarrow 0$ as $\omega_{q} \rightarrow \omega_{0}$. This procedure is consistent with the interaction spectrum obtained from tunneling experiments. The significance of the four terms on the right hand side of equation (6) has been explained in reference 10. We will plot $f\left(\omega, \omega_{0}, T_{c}\right)$ as a function of $T_{c} / \omega_{0}$ and $\omega / \omega_{0}$ obtained from numerical results in section III.

We evaluate $\Delta^{2}(0)$ by retaining the Bose factor but without neglecting $\omega_{q}$ compared with $\omega$, that is

$$
\begin{aligned}
\Delta_{0}^{2}= & \frac{\Delta_{\infty}}{Z_{0}} \int_{\omega_{0}}^{\infty} \frac{d \omega^{\prime}}{\omega^{\prime}} \int_{0}^{\omega_{0}} d \omega_{q} \alpha^{2} F\left(\omega_{q}\right) \frac{2}{\omega^{\prime}+\omega_{q}} \\
& +\frac{4 \Delta_{\infty}}{Z_{0}} \int_{0}^{\omega_{0}} \frac{d \omega_{q}}{\omega_{0}} \alpha^{2} F\left(\omega_{q}\right) N\left(\omega_{q}\right)
\end{aligned}
$$


Equation (7) is shown in the Appendix that it can be approximated by

$$
\Delta_{0}^{2} \cong \frac{\Delta_{\infty}}{Z_{0}}\left\{\lambda \ln \left(1+\frac{\langle\omega\rangle}{\omega_{0}}\right)+4 \int_{0}^{2.5 T_{c}} \frac{d \omega_{q}}{\omega_{0}} \alpha^{2} F\left(\omega_{q}\right) N\left(\omega_{q}\right)\right\}
$$

For $\frac{\langle\omega}{\left.\omega_{0}\right\rangle} \sim 0.6$, the first term on the right hand side of equation (8) differs from the previous result of McMillan by $\sim 20 \%$. It has the effect of reducing the repulsion due to electronic interaction with frequency components greater than $\omega_{0}$. The second term can be calculated by assuming a power law dependence for $\alpha^{2} F\left(\omega_{q}\right)$ on $\omega_{q}$ in the form

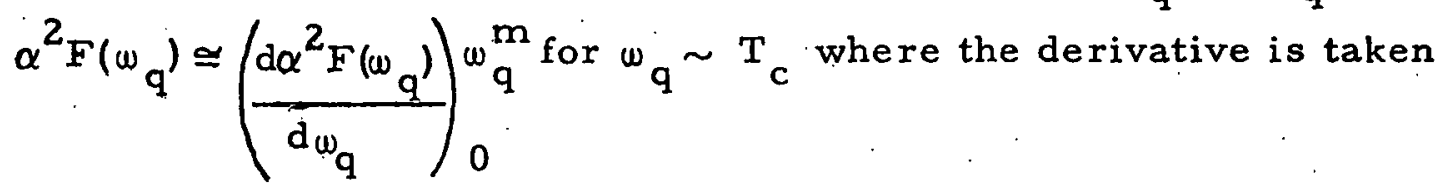
arbitrarily close to $w_{q}=0$, and $m$ has been observed from tunneling experiments to be of order unity for strong-coupled superconductors. For weak-coupled superconductors (i.e. with the cut off frequency at the lower end of the interaction spectrum greater than $\sim 3 T_{c}$ ), this term can be dropped. In general, it is shown in the Appendix that this small correction term has a $\mathrm{T}_{c}^{\mathrm{m}+1}$ dependence on $\mathrm{T}_{\mathrm{c}} \cdot$. Combining these two terms, $\Delta^{2}(0)$ can be written as

$$
\Delta_{0}^{2} \cong \frac{\Delta_{\infty}}{Z_{0}}\left\{\lambda \ln \left(1+\frac{\langle\omega\rangle}{\omega_{0}}\right)+E(m)\left(\frac{d \alpha^{2} F(\omega)}{d \omega}\right) \frac{T_{c}^{m+1}}{\omega_{0}}\right\}
$$

where $E(m)=\frac{4}{m}\left[\frac{1}{2}+\frac{2^{m-1}}{3}+\frac{1}{3}\left(\frac{5}{2}\right)^{m}\right]$

$\Delta^{2}(0)$ is an increasing function of $T_{c}$ due to the inclusion of thermal phonons in the self-consistent solution of the model chosen. For 
$\mathrm{m} \sim 1, \mathrm{~T}_{\mathrm{c}} \sim 0.1 \omega_{0}$; the temperature correction term is approximately equal to $6 \%$ of the first term on the right hand side of equation (9). Thus it can be dropped for $T_{c} \lesssim 0.1 \omega_{0}$. However, for $T_{c}$ beyond this range, it certainly has a repulsive effect on $\Delta(0)$ and thus a diminished effect on $T_{C}$ as can be seen later.

The electron-phonon mass enhancement factor $Z_{0}$ evaluated at $\mathbf{T}_{\mathbf{c}}$ due to thermal smearing of the Fermi Surface has been obtained numerically by Allen. 12 The result for the case of a spherical Fermi surface takes the form

$$
Z_{0}\left(T_{c}\right)=1+\lambda_{m} \cong 1+\lambda\left[1+3 \cdot 5\left(T_{c} / \omega_{0}\right)^{2}\right]
$$

Finally, as shown by McMillan ${ }^{5}$, the above equations can be grouped into two linear homogeneous equations. The condition for the existence of non-vanishing solutions to $\Delta_{0}$ and $\Delta_{\infty}$ at $T_{c}$ yields an expression for $\mathbf{T}_{\mathbf{c}}$

$$
T_{c}=1.134 \omega_{0} \cdot \exp \left\{-\frac{1+\lambda_{m}+2 \int_{0}^{\omega_{0}} \frac{d \omega}{\omega} \alpha^{2} F(\omega) f\left(\omega, \omega_{0}, T_{c}\right)}{\lambda-\mu^{*}\left(1+\lambda \ln \left(1+\frac{\langle\omega\rangle}{\omega_{0}}\right)\right)}\right\}
$$

Expression (11) can be compared to McMillan's equation for $T_{c}$ given by 5

$$
T_{c}=1: 134 \omega_{0} \exp \left\{-\frac{1+\lambda}{\lambda-\mu^{*}\left(1+\lambda \frac{\langle\omega\rangle}{\omega_{0}}\right)}\right\}
$$

Our expression for $T_{c}$ is an implicit one in which $T_{c}$ appears in the exponential term which has the effect of restricting $T_{c} / \omega_{0}$ to a limit compatible with experimental results as can be shown in later sections. 


\section{NUMERICAL SOLUTIONS}

Expression (1) in section II is an exact solution to the Eliashberg equations at $T=T_{c}$ for the gap model chosen. In order to exploit the physical implications inherent in this expression, we have to look for a way of transforming the integral term inside the exponential into a more convenient form. Certain approximations will be made to yield a new expression for $T_{c}$ in terms of the materials parameters $\lambda, \mu^{*}$ and $\langle\omega\rangle$. We first plot $f\left(\omega, \omega_{0}, T_{c}\right)$ as a function of $\omega / \omega_{0}$ for different constant values of $\mathrm{T}_{\mathrm{c}} / \omega_{0}$. Four representative values of $\mathrm{T}_{\mathrm{c}} / \omega_{0}$ are chosen in Fig. 1. The shapes of these curves suggest that they might be approximated by a power function of $\omega / \omega_{0}$ in the form.

$$
f\left(\omega, \omega_{0}, T_{c}\right) \cong f_{1}\left(\frac{T_{c}}{\omega_{0}}\right)-g\left(\frac{\omega}{\omega_{0}}\right)^{n}
$$

where $f_{1}\left(\frac{T_{c}}{\omega_{0}}\right)=\lim _{\omega \rightarrow 0} f\left(\omega, \omega, T_{c}\right)=\ln \left(\frac{1.134 \omega_{0}}{T_{c}}\right)+\frac{2 T_{c}}{\omega_{0}}$

$g=g\left(\frac{T}{\omega_{0}}\right)$ and $n=n\left(\frac{T}{c}\right)$ are functions of $\frac{T_{c}}{\omega_{0}} \cdot$ It is interesting to

to note that all curves converge to $\sim 0.7$ as $\omega \rightarrow \omega_{0}$. In fact, it can be shown that $f\left(\omega_{0}, \omega_{0}, T_{c}\right) \cong \ln 2+\left(\frac{2 T}{\omega_{0}}\right)^{2}$

The power functions which fit the numerical curves best are plotted as dotted lines in Fig. 1 for comparison with numerical results of $f\left(w, \omega_{0}, T_{c}\right)$. We try to minimize the errors of our plots between. $\frac{\omega}{\omega_{0}}=0.2$ and 0.9 . The numbers $\mathrm{g}$ and $\mathrm{n}$ for different values of $\mathrm{T}_{\mathrm{c}} / \omega_{0}$ are obtained graphically from $f_{1}\left(\frac{T c}{\omega_{0}}\right)-f\left(\omega_{0}, \omega_{0}, T_{c}\right)$ and the slopes of 


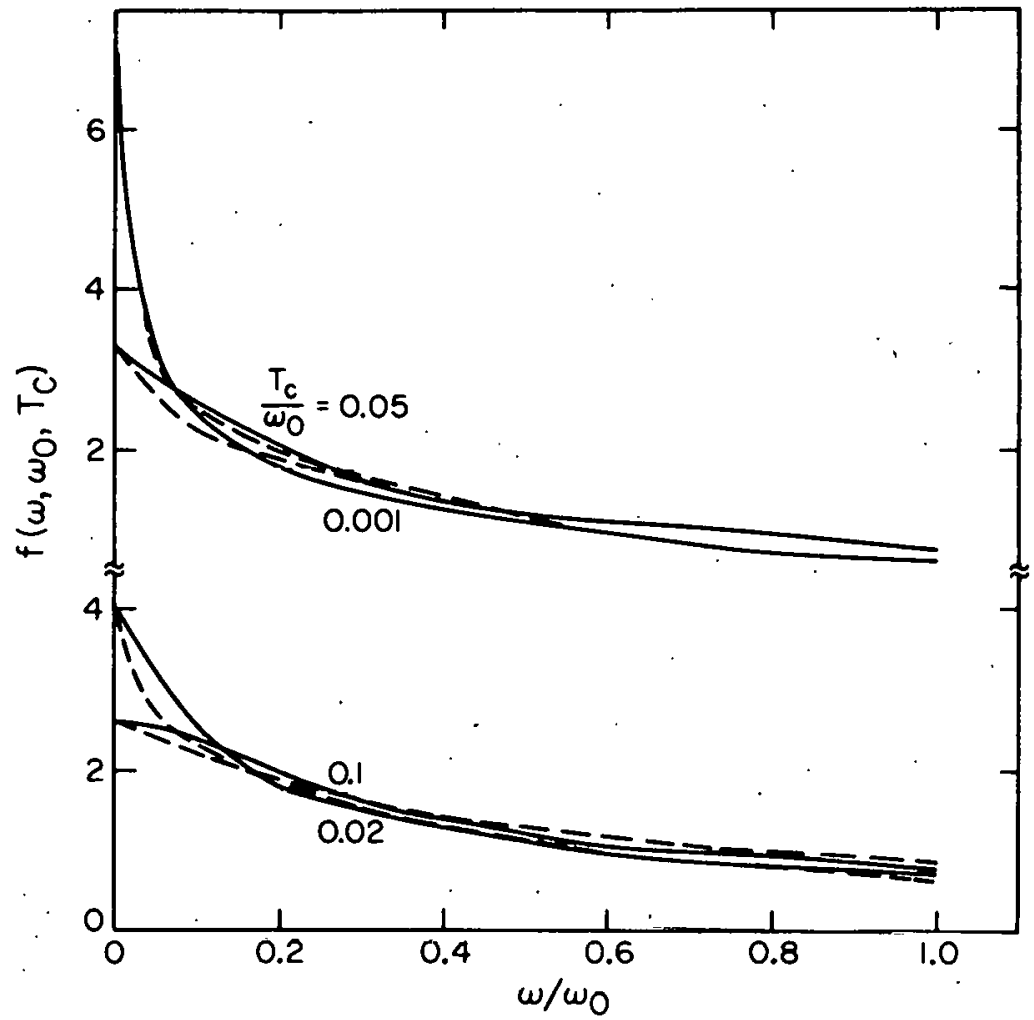

Fig. 1. Plots of $\mathrm{f}\left(\omega_{0}, \mathrm{~T}_{c}\right)$ as a function of $\omega_{0} / \omega_{0}$. Solid lines denote numerical results obtained from equation (6). Dashed lines are obtained from power law fittings as shown in Fig. 2. 
curves on the log-log plot respectively. Portions of the fitting functions are plotted on a $\log -\log$ scale in Fig. 2 together with data points obtained numerically from equation (6). Besides the representative curves, more numerical curves and fitting functions have been plotted to yield $g$ and $n$ as continuous functions of $T_{c} / \omega_{0} \cdot$ They are plotted together with the data points in Fig. 3. It should be noted that $g \rightarrow \ln \left(\frac{1.134 \omega_{0}}{T_{c}}\right)$ as $\frac{T_{c}}{\omega_{0}} \rightarrow 0 . A \ln \left(\frac{1.134 \omega_{0}}{T_{c}}\right)$ curve is plotted on the same figure for comparison. This fact will be used later to yield an expression for $T_{c}$ for weak-coupled superconductors. The value of $n$ is less than $\sim 0.5$ in the range of $\mathrm{T}_{\mathrm{c}} / \omega_{0}$ considered.

Substituting the power function $f\left(w, w_{0}, T_{c}\right)$ in equation (13) obtained from power law approximations into our earlier expression for $T_{c}$ in equation (11), we obtain

$$
\begin{aligned}
T_{c}=1.134 \omega_{0} \exp \left\{-\frac{1}{-} \frac{\lambda_{m}+\left[\ln \left(\frac{1.134 \omega_{0}}{T_{c}}\right)+\frac{2 T_{c}}{\omega_{0}}\right] \lambda}{\lambda-\mu^{*}\left[1+\lambda \ln \left(1+\frac{\langle\omega\rangle}{\omega_{0}}\right)\right]}\right. \\
\left.+\frac{\left(2 g / \omega_{0}^{n}\right) \int_{0}^{\omega_{0}} \frac{d \omega}{\omega^{1-n}} \alpha^{2} F(\omega)}{\lambda-\mu^{*}\left[1+\lambda \ln \left(1+\frac{\langle\omega\rangle}{\omega_{0}}\right)\right]}\right\}
\end{aligned}
$$

So far, our approximations involved in arriving at equation (14) have been reasonable. We can go ahead and simplify this equation further by employing another assumption which involves the way one would average frequency over an electron-phonon interaction spectrum. 


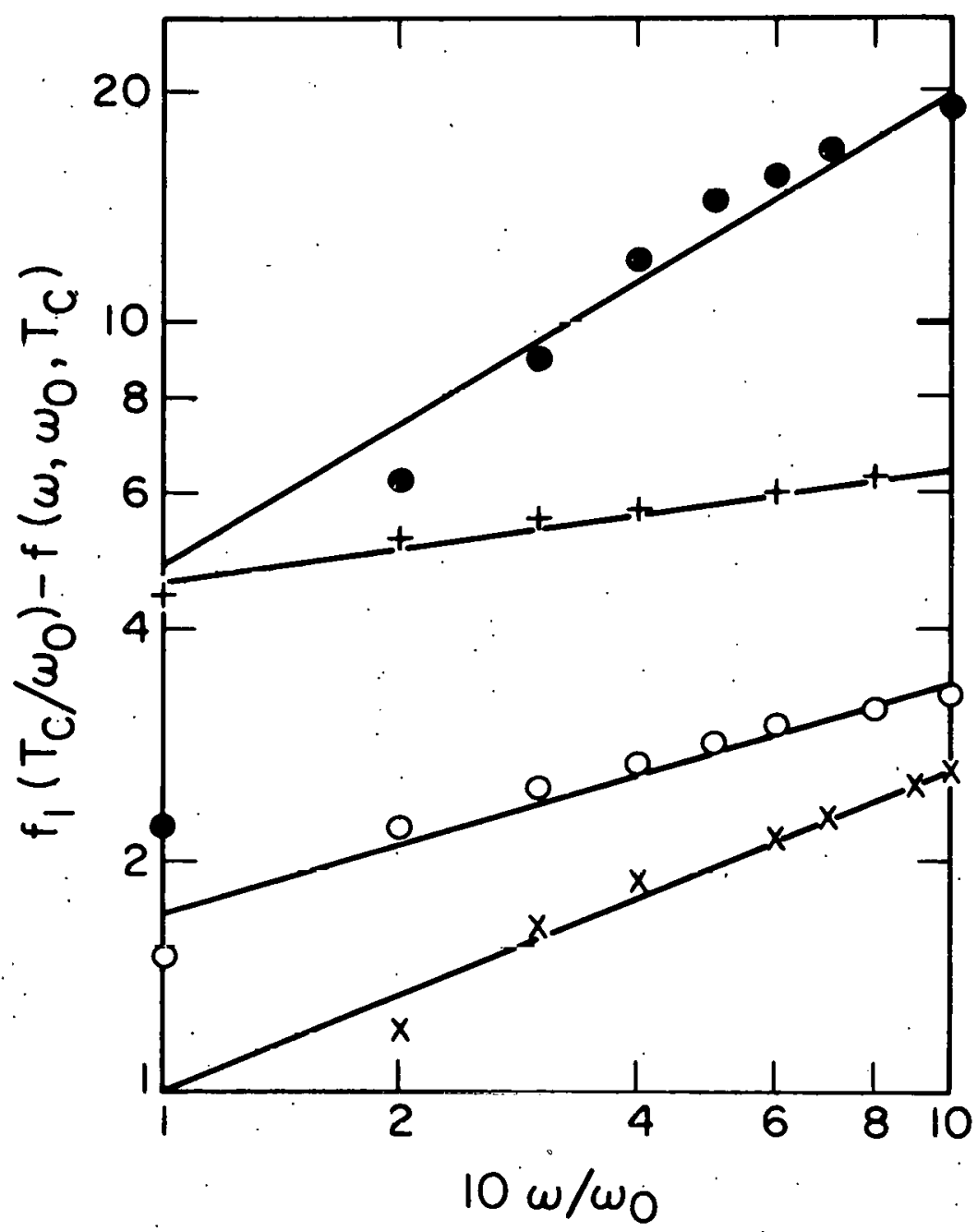

Fig. 2: Sketch for power function fittings as defined in equation (13). Straight lines give dashed lines in Fig. 1. Data points obtained from equation (6) represent: $e, x, 0$, and $t$ are for $\frac{T_{c}}{\omega_{0}}=0.1$, $0.05,0.02$, and 0.001 respectively. 


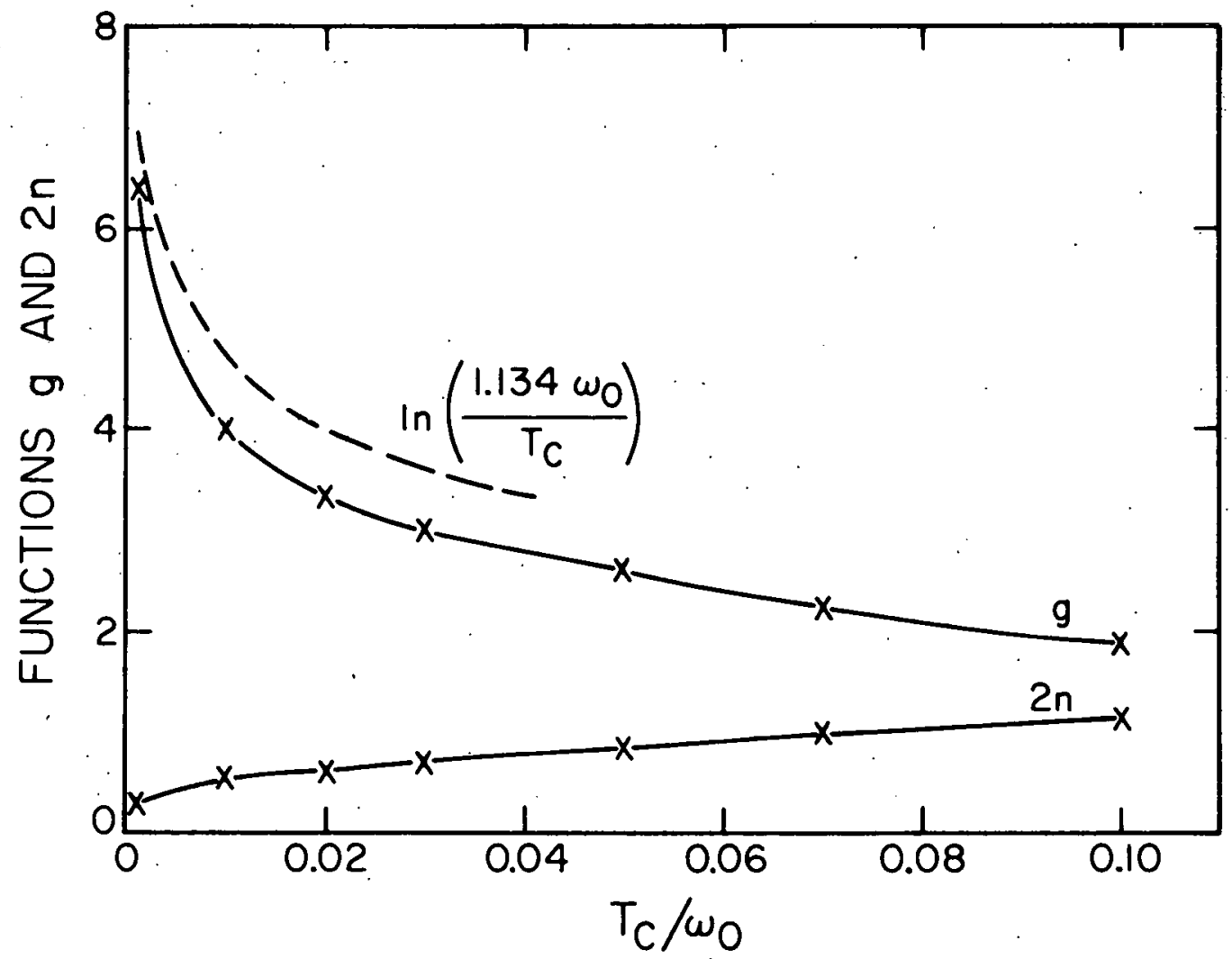

Fig. 3. Plots of $g$ and $2 n$ as a function of $T_{c} / \omega_{0}$, where $g$ and $n$ are functions defined in equation (18). 
We assume

$$
\left[\frac{\int_{0}^{\omega_{0}} \frac{\alpha^{2} F(\omega)}{\omega^{1-n} d \omega}}{\int_{0}^{\omega_{0}} \alpha^{2} F(\omega) d \omega}=\frac{\int_{0}^{\frac{1}{1-n}} \frac{\alpha^{2} F(\omega)}{\omega} d \omega}{\int_{0}^{\omega_{0}} \alpha^{2} F(\omega) d \omega}\right.
$$

where $\mathrm{n} \lessgtr 0.5$ as mentioned above. Intuitively, we would expect this to be quite a reasonable assumption. For the sake of accuracy, we would like to check relation (15) by computing the averages involved for both the strong-coupled and weak-coupled interaction spectra. We use the $\mathrm{Nb}_{3} \mathrm{Sn}^{13}$ and amorphous $\mathrm{BiPb}^{14}$ spectra for the strong-coupled case, and $\mathrm{Nb}^{5}$ for the weak-coupled case. Actually, the only case to worry about is the strong-coupled case. These are typical spectra for the corresponding cases. It turns out that the errors in these averages are within $8 \%$ and thus relation (15) is well established for both classes of materials. We can rewrite this relation in the form

$$
\begin{aligned}
\int_{0}^{\omega_{0}} \frac{\alpha^{2} F(\omega)}{\omega^{1-n}} d \omega & =\left[\int_{0}^{\omega_{0}} \frac{\alpha^{2} F(\omega)}{\omega} d \omega\right]^{1-n}\left[\int_{0}^{\omega_{0}} \alpha^{2} F(\omega) d \omega\right]^{n} \\
& =\frac{\lambda\langle\omega\rangle^{n}}{2}
\end{aligned}
$$

where the definition $\lambda\langle\omega\rangle=2 \int_{0}^{\omega_{0}} \alpha^{2} F(\omega) d_{\omega}$ has been used.

We substitute equation (16) into equation (14) and then simplify the expression by taking out the $\ln \left(\frac{1.134 \omega_{0}}{T_{c}}\right)$ term from the exponential term. A completely new expression for $T_{c}$ is obtained 


$$
T_{c}=1.134 \omega_{0} \exp \left\{\frac{1+\lambda_{m}+\left[\frac{2 T_{c}}{\omega_{0}}-g\left(\frac{\langle\omega\rangle}{\omega_{0}}\right)^{n}\right] \lambda}{\mu^{*}\left(1+\lambda \ln \left(1+\frac{\langle\omega\rangle}{\omega_{0}}\right)\right)}\right\}
$$

For the two-square-well problem, this is our central result which is general. Equation (17) can be regarded as a "graphical solution" to expression (11) without loss of generality (at most within a few percent error 1. The curve fittings have been a materials independent procedure. For weak coupling (take $\left.T_{c} \lesssim 0.02 \omega_{0}\right), g\left(\frac{T_{c}}{\omega_{0}}\right) \rightarrow \ln \left(\frac{1.134 \omega_{0}}{T_{c}}\right)$, $\underset{\omega_{0}}{\langle\underline{\omega}\rangle} \geqslant 0.7$, and $\left(\frac{\underline{\omega}\rangle}{\omega_{0}}\right)^{\mathrm{n}} \sim 1$, we obtain $\mathrm{T}_{\mathrm{c}} \cong 1.134 \omega_{0} \exp \left\{-\frac{1+\lambda}{\lambda-\mu^{*}\left(1+\lambda \ln \left(1+\frac{(\omega)}{\omega_{0}}\right)\right)}\right\}$ which is just the McMillan equation for $T_{c}$. The minimum $\lambda$ for the occurrence of superconductivity is thus $\lambda_{\min }=\frac{\mu}{1-\mu^{*} \ln \left[1+\frac{\langle\underline{\omega}\rangle}{\omega_{0}}\right]}<\mu$, a macroscopic stability requirement for the lattice without including the effects of umklapp scattering in $\lambda$.

IV. FITTING $T_{c}$ EXPRESSION TO TUNNELING DATA.

Equation (17) is correct to within several percent error using the two-square-well model. The gap function $\Delta(\omega)$ for different superconductors determined from tunneling experiments at $T<\leqslant T_{c}$ but iterated at $T=0$ give results quite different from the model we choose. Although at $T \cong T_{c}$, the thermal smearing effect on $\Delta(\omega)$ will compensate for this disagreement, yet one has to solve both $\operatorname{Re} \Delta(\omega)$ and $\operatorname{Im} \Delta(\omega)$ self-consistently from the Eliashberg equations in order to obtain the correct $T_{c}$. Therefore, one should not expect equation (17) 
to yield exact values of $T_{c} / \omega_{0}$ once the materials parameters $\mu^{*}, \lambda$, and $\langle\omega\rangle / \omega_{0}$ are given. Instead, our intention has been to derive an expression which gives a more restricted value of $T_{c} / \omega_{0}$ than that given by the McMillan equation. Then, we proceed to improve equation (17) for self-consistency by replacing the premultiplier $\omega_{0}$ by a mean phonon frequency $\bar{\omega}=\int_{0}^{\omega} \omega \alpha^{2} F(\omega) \mathrm{d} \omega / \int_{0}^{\omega_{0}} \alpha^{2} F(\omega) \mathrm{d} \omega=\left\langle\omega^{2}\right\rangle /\langle\omega\rangle$ as first used by Dynes. ${ }^{15}$ This is a reasonable value to use because the $\omega_{0}$ in a self-consistent solution clearly depends on the details of the electron-phonon interaction spectrum. We first plot $\lambda$ versus $\left.1.134 \mathrm{~h}\left(\lambda, \mu^{*}, \underline{\omega}_{0}\right\rangle\right)$ in Figs, $4(\mathrm{a}\rangle$ and $4(\mathrm{~b})$, where $\mathrm{h}$ stands for the exponential function in equation (17). The variable $T_{c} / \omega_{0}$ has been dropped from $h$ because it is determined implicitly from the other variables. It can be seen from Fig. 4 that equation (17) gives more restricted values of $\mathrm{T}_{c} / \omega_{0}$ than those obtained from tunneling experiments. This is probably due to the inconsistency of the solutions as explained above.

We proceed to improve the self-consistency of our solutions by fitting equation (17) to tunneling data. A plot of $\left\langle\omega^{2}\right\rangle / 10\langle\omega\rangle T_{c}$ versus $\left[11.34 \mathrm{~h}\left(\lambda, \mu^{*}, \frac{\langle\omega\rangle}{\omega_{0}}\right)\right]^{-1}$ for eleven alloys is given in Fig. 5. The alloys and their relevent parameters determined from tunneling experiments are given in Table I. This data contains a wide variety of materials and phonon spectra including amorphous thin films, disordered alloys, soft and transition metals, and $\beta-W \mathrm{Nb}_{3} \mathrm{Sn}$, with $\lambda$ ranging from 0.69 to 3.3. It represents an up to date account of tunneling results. A well defined straight line with gradient equal to 0.4 is obtained. Thus we rewrite equation (17) as

$$
T_{c}=2.835 \bar{\omega} h\left(\lambda, \mu^{*}, \frac{\langle\omega}{\omega_{0}}\right)
$$




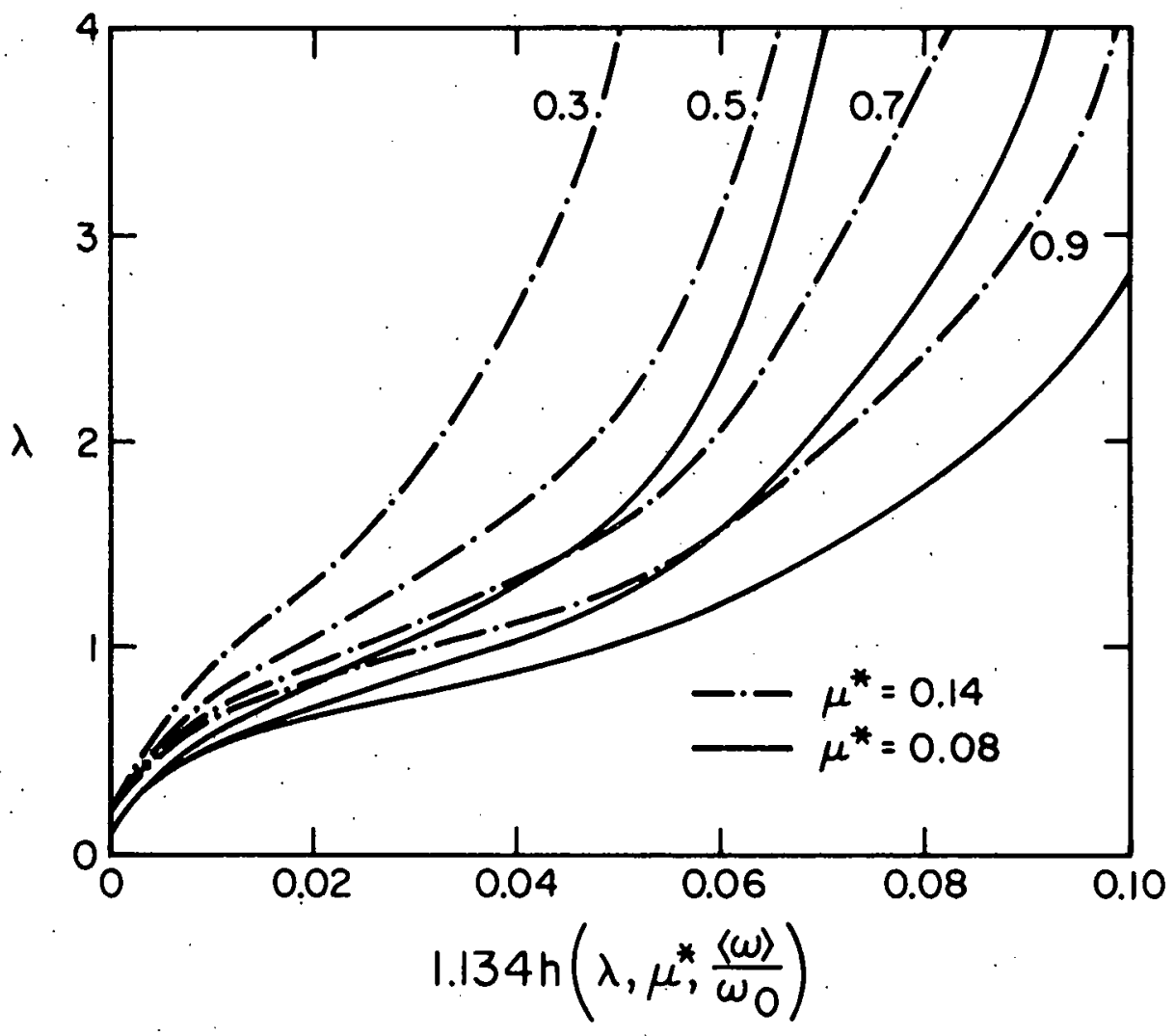

Fig. 4. (a) Plots of $\lambda$ versus $1.134 \mathrm{~h}\left(\lambda, \mu^{*}, \frac{\langle\omega}{\omega_{0}}\right)$. The number associated with each curve denotes the value of ${\stackrel{\omega \omega}{\omega_{0}}}_{0}$. 


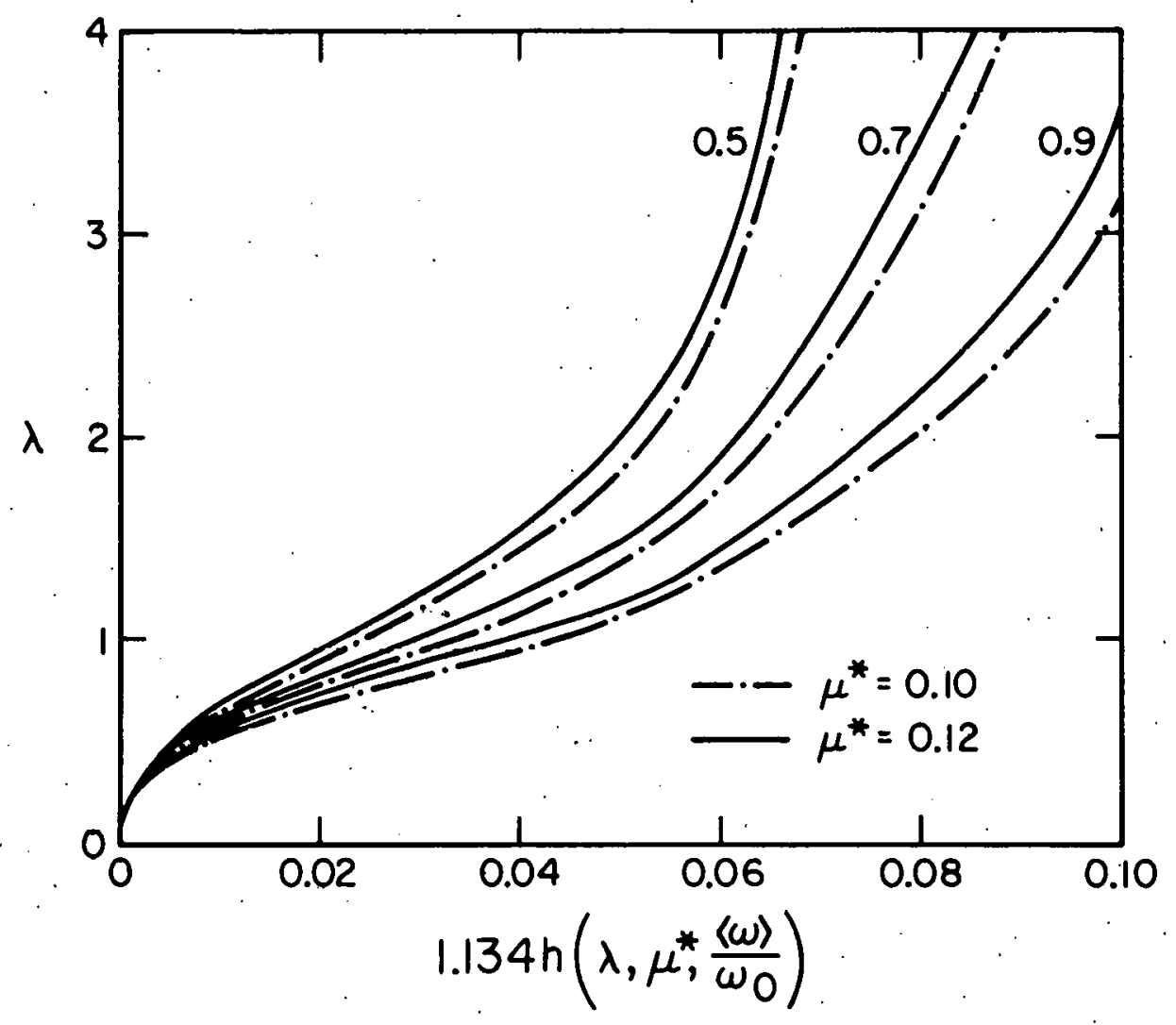

Fig. 4.(b) Same as Fig. 4(a) for different $\mu^{*}$. 
Table I. Relevant parameters obtained from tunneling experiments for Fig. 5 .

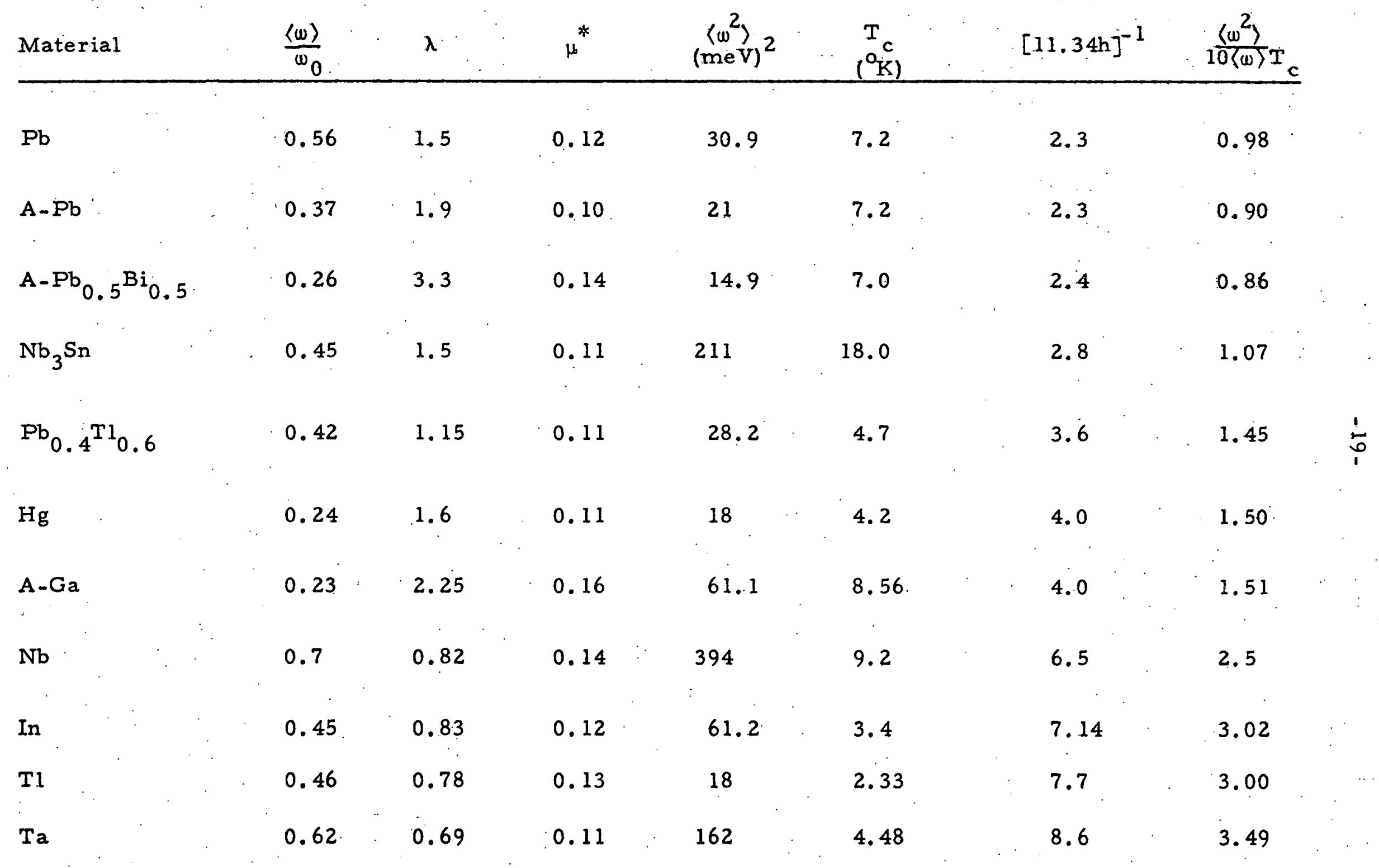

The data are obtained from references $5,13,14,16,17,18,19$, and 20 . $A$ - amorphous 


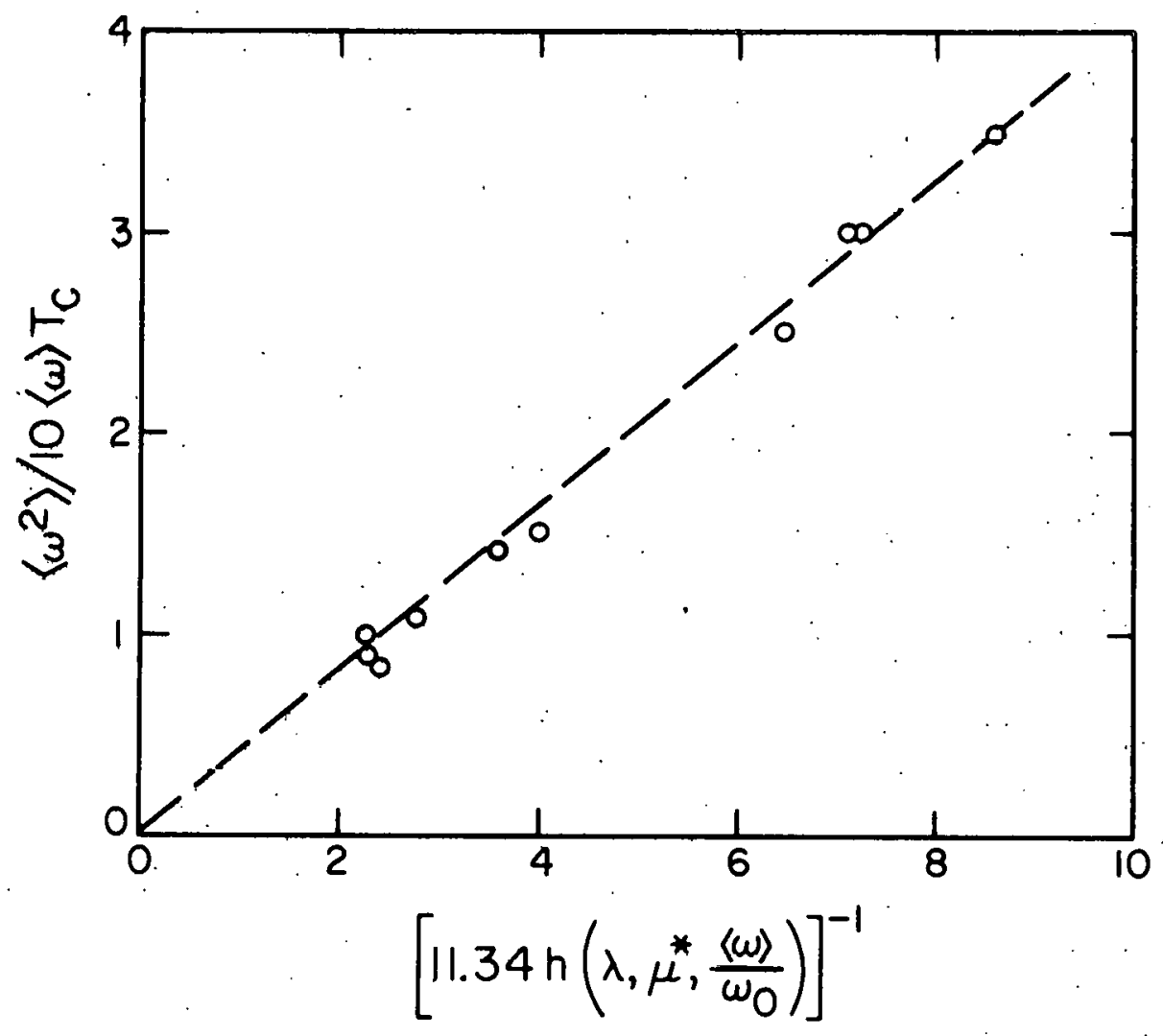

Fig. 5. Piots of. $\frac{\left\langle\omega^{2}\right\rangle}{10\langle\omega\rangle \mathrm{T}_{c}}$ versus $\left[11.34 \mathrm{~h}\left(\lambda, \mu^{*}, \frac{\langle\omega}{\omega_{0}}\right]^{-1}\right.$ for the eleven superconductors given in Table. I. 
Equation (18) is our final expression for $T_{c}$ which is believed to be general to within $15 \%$ error, One should also note that the tunneling data used here are obtained experimentally at $T \neq 0$ but determined mathematically at $T=0$. Hence, one should not demand equation (18) to yield exact $T_{c}^{\prime} / s$ beyond its self-consistency. Using the modified McMillan equation as introduced by Dynes ${ }^{15}$ and rewriting it in the form of equation (18), one always obtains higher values of $h$ for $\frac{\langle\omega}{\omega_{0}} \leqslant 0.6$.

\section{SUPERCONDUCTIVE MATERIALS}

We investigate in this section the superconductivity in various classes of materials based on equation (18). We first concentrate on the existing high $T_{c}$ alloys, then apply our present stage of knowledge to other prospective systems. The maximum $\mathbf{T}_{\mathbf{c}}$ in various systems will be discussed. These maxima are definitely limited by the maximum electron-phonon coupling constant $\lambda$ attainable in real systems. From Table $I$, it is observed that alloys with small $\langle\omega\rangle / \omega_{0}$ tend to give large $\lambda$. This fact seems to be rather general even for alloys not listed in the Table. Referring to Fig. 4, the first derivative $\frac{\mathrm{d} \lambda}{\mathrm{dh}}$ for given $\left.\underset{\omega_{0}}{\langle\omega}\right\rangle$ and $\mu$ * increases more rapidly after some critical value $\lambda_{c} \cdot$ Higher increment of $\lambda$ is required to enhance $T_{c}$ beyond that given by $\lambda_{c}$. In Table II, approximate values of $\lambda_{c}$ for different $\frac{\langle\omega\rangle}{\omega_{0}}$ and $\mu^{*}$ are given. It is observed that values of $\lambda$ and other parameters given in Table I and II are consistent (i.e. $\lambda<\lambda_{c}$ ). Thus $\lambda_{c}$ is probably a reasonable approximation to use when the maximum $T_{c}$ in a system is considered.

(1) In reference 11 , the systematics of superconductivity in transition metal (TM) alloys have been discussed empirically. It was 
Table II. Values of the critical coupling constant $\lambda_{c}$ for various $\mu^{*}$ and $\frac{\langle\omega}{\omega}{ }_{0}$ as defined in the text.

$\frac{(m))_{0}}{\omega_{0}^{*}}$
ก. 08
0.1
0.12
0.14

0.3

$>2$

$>2$

$>2$

$>2$

0.5

1.6

1.6

1.75

1.75

0.7

1,3

1.4

1.5

1.6

0.9

1.1

1.2

1.25

1.3 
concluded that the $T \dot{M}$ alloys with the highest $T_{c}$ should occur in the Mo-Tc system. A plot of $N\left\langle I^{2}\right\rangle / q_{0}^{2}$ versus melting temperature $T_{m}$ was obtained from the coupling constants $\lambda$ evaluated using the McMillan $T_{c}$ equation. A different scheme of $\lambda$ is expected if equation (18) is used instead. Since the alloys given in the plot are mostly $\beta-W$ compounds, we assume $\mu^{*} \sim 0.12, \frac{\langle\omega\rangle}{\omega_{0}} \sim 0.5$, and $\bar{\omega} \sim 0.6 \theta_{\mathrm{D}}$ which are reasonable values for $\beta-W$ spectra (e.g. $\mathrm{Nb}_{3} \mathrm{Sn}$ ). Equation (18) can then be approximated by the formula $T_{c} \cong 1.69 \theta_{D}$ h. Values of $\lambda$ obtained this way vary from 0.95 to 1.45 as compared to the previous values of 0.8 to 1.1. Both old and new plots of $N\left\langle I^{2}\right\rangle / q_{0}^{2}$ versus $T_{m}$ are shown in Fig. 6. The new scheme also yields a straight line although it is based on our new equation (18). It should be emphasized again that the actual values in Fig. 6.are not expected to be exact because of the way $\mathrm{N}\left\langle\mathrm{I}^{2}\right\rangle / \mathrm{q}_{0}^{2}$ is computed from $\lambda$. Instead, its systematic behavior as a function of $\mathrm{T}_{\mathrm{m}}$ is most important. For Mo-Tc alloys, the value of $\lambda$ is 1.3 based on $T_{c} \sim 16^{\circ} \mathrm{K}$. A complete phase diagram of Mo-Tc does not exist. However, if the melting temperature of an Mo-Tc alloy satisfying the 6.5 valence electrons rule is higher than $\sim 2250^{\circ} \mathrm{K}$ as shown in Fig. 6, a $\mathrm{T}_{\mathrm{C}}>16^{\mathrm{O}} \mathrm{K}$ can be obtained from a higher $\lambda$ through an increased $N\left\langle I^{2}\right\rangle$. The 5-d alloys always give higher $N\left\langle I^{2}\right\rangle$ but smaller $\lambda$ through an increased atomic mass $M$.

(2) For $\beta-W$ compounds of the form $\mathrm{Nb}_{3} \mathrm{X}$ and $\mathrm{V}_{3} \mathrm{X}$, where $\mathrm{X}$ stands for a group A element, we examine the maximum $T_{c}$ when the $\lambda_{c}=2$ condition is reached keeping other parameters fixed. From Fig. 4, values of 0.043 to 0.045 for $h$ are obtained given $\langle\underline{\omega}\rangle \sim 0.45$ to 0.5 and $\mu^{*} \sim 0.11$. This gives $T_{c} \cong 0.121$ to $0.129 \bar{\omega}$. If ${ }_{a}^{\omega}$ value of $\sigma=16.6 \mathrm{meV}$ as for $\mathrm{Nb}_{3} \mathrm{Sn}$ is taken, then $\mathrm{T}_{\mathrm{c}}^{\max } \cong 23.3$ to $25^{\circ} \mathrm{K}$ is obtained. This 


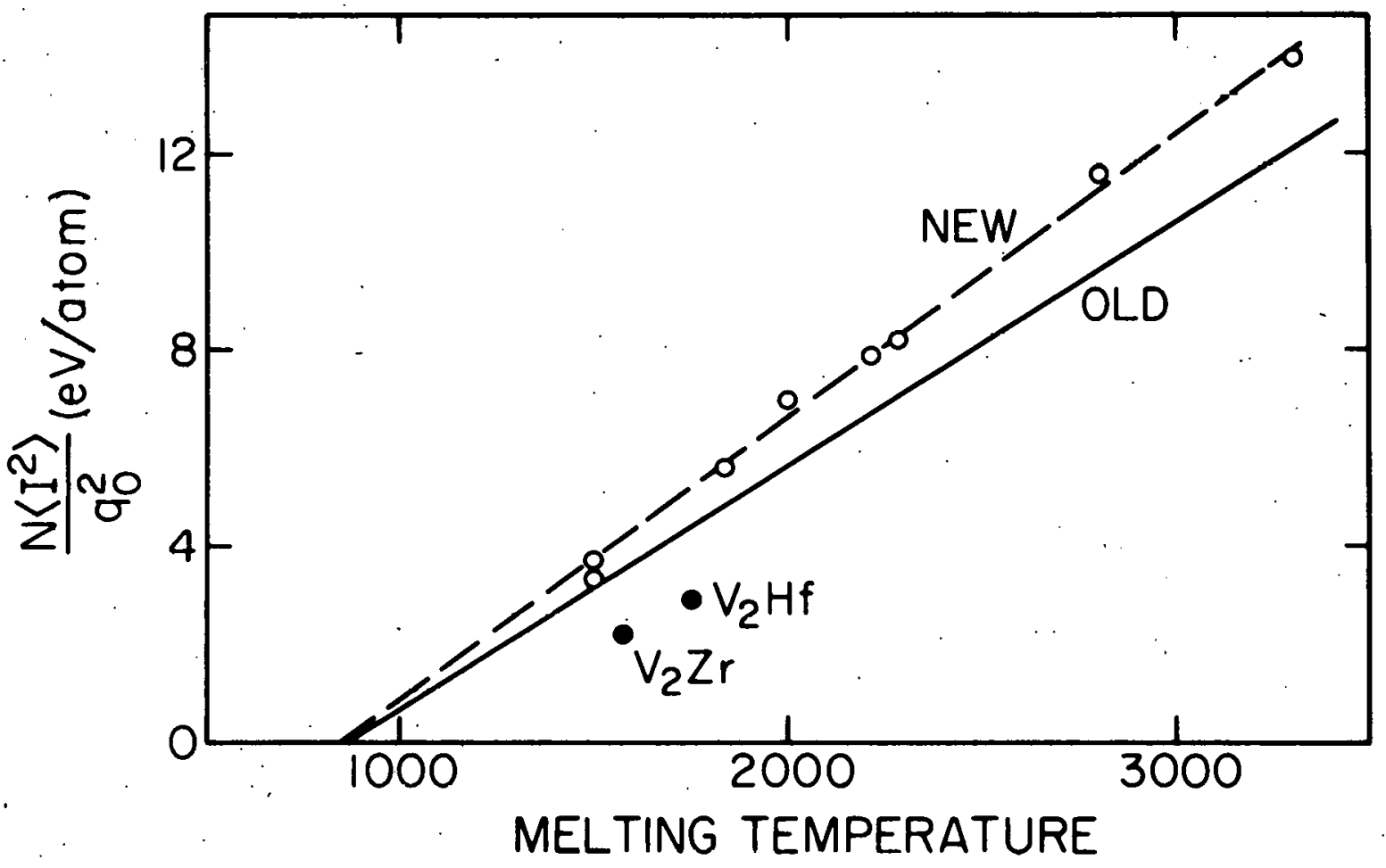

Fig. 6. Values of $\frac{N(0)\left\langle I^{2}\right\rangle}{q_{0}{ }^{2}}$ as.a function of $T_{m}$ for $4 d$ and $5 d$ alloys. Solid line denotes the curve given in Poon and Johnson's paper. Dashed line denotes values obtained using equation (18). Two 3d alloys are also given. 
demonstrates the inherent limitation on $\mathrm{T}_{c}$ as implied by equation (18). For the $V_{3} X$ alloys, the values of $T_{c}$ a re even more limited than the $\mathrm{Nb}_{3} \mathrm{X}$ alloys through reduced $\lambda$ 's as discussed in our previous paper. We believe that $\mathrm{T}_{\mathrm{c}} \sim 25^{\circ} \mathrm{K}$ will be the limit for $\mathrm{Nb}_{3} \mathrm{X}$ alloys as discussed before. $^{11}$ If instead of equation (18), we use the McMillan equation but replace its premultiplier by $\bar{\omega} / 1.2$ as used by Dynes ${ }^{15}$ to predict $\mathrm{T}_{\mathrm{c}}^{\mathrm{max}}$, a value of $\sim 30^{\circ} \mathrm{K}$ is obtained.

(3) No tunneling data are available for the high $T_{c}$ nitrides and carbides. Phillips ${ }^{21}$ estimated on the basis of McMillan equation with $\mu^{*}=0$ that for $\mathrm{NbN},\left\langle\omega^{2}\right\rangle^{\frac{1}{2}} \sim 150^{\circ} \mathrm{K}$, and $\mathrm{T}_{\mathrm{c}}^{\max }$ for this class of materials to be $22^{\circ} \mathrm{K}$. If we take $\mu^{*}=0, \underline{\omega}_{\omega_{0}}^{\left\langle\omega^{\prime}\right.} \sim 0.5$ based on $\left\langle\omega^{2}\right\rangle^{\frac{2}{2}}=150^{\circ} \mathrm{K}$ and $\theta_{\mathrm{D}}=250^{\circ} \mathrm{K}$ for $\mathrm{NbN}$, equation (18) predicts $\lambda_{\mathrm{c}} \sim 1.25$ and $\mathrm{h} \sim 0.0467$. A value of $\bar{\omega} \sim 0.65 \theta_{\mathrm{D}}=160^{\circ} \mathrm{K}$ then gives $\mathrm{T}_{\mathrm{c}}^{\mathrm{max}} \sim 21^{\circ} \mathrm{K}$. In reality, $\mu^{*} \neq 0$ and one can take $\mu^{*} \sim 0.11$ as for most niobium compounds, values of $\lambda_{c} \sim 1.75$ and $h \sim 0.0414$ are obtained. This estimate gives $T_{c}^{\max } \sim 19^{\circ} \mathrm{K}$ instead. Although, the real $T_{c}^{\max }$ for the nitrides and carbides is expected to be in reasonable agreement with our estimates, yet experimental determination of $\alpha^{2} F(\omega)$ and $\mu^{*}$ for this class of materials is by. itself interesting and informative.

(4) We examine the prospects of obtaining high $T_{c}$ in materials with a strong tendency towards forming bonds. This class of materials has been discussed by Anders on and Cohen. ${ }^{8}$ It was pointed out that umklapp scattering can lead to strong electron-phonon coupling and thus results in lattice instability. We consider before different classes of

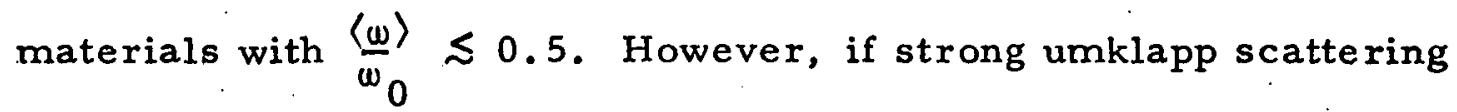
(i.e. scattering with large $|\vec{q}|$ is present in the lattice, one expects $\frac{\langle\omega\rangle}{\omega_{0}}$ to go up simultaneously with an increased $\omega_{0}$ in covalent like 
materials. Say, if $\underline{\omega}_{0} \underline{\omega}_{0}$ is taken to be 0.7 and $\mu^{*} \sim 0.12$, values of $\lambda_{c} \sim 1.5$ and $h \sim 0.044$ are obtained. This gives $T_{c}^{\max } \sim 0.125 \bar{\omega} \sim 0.09 \theta_{D^{\prime}}$. If an extreme value of $\frac{\langle\omega}{\omega_{0}} \sim .0 .9$ is taken for the case of a $\delta$-function peak at $\omega_{0}$, a similar value of $T_{c}^{\max }$ is obtained through a decreased $\lambda_{c} \sim 1.25$. We believe that such $T_{c}^{\max }$ is realistic because the $\lambda_{c}$ is are attainable based on previous expermental results, A value of $\theta_{\mathrm{D}} \sim 400^{\circ} \mathrm{K}$ is probably high enough for a covalent like material and thus $\mathrm{T}_{\mathrm{C}}^{\max }$ can be as high as $36^{\circ} \mathrm{K}$.

A rather clear correlation can be seen from the above discussion between the values of $\frac{\langle\omega}{\omega_{0}}$ and $h$, and that $T_{c}^{\max }$ is mainly determined by the mean phonon frequency $w$. In fact, it is observed in Table II that the product $\lambda_{c} \underset{\omega_{0}}{\left\langle\omega_{0}\right\rangle}$ decreasesslowly as the parameter $\frac{\langle\omega \omega}{\omega_{0}}$ decreases. The exponential term $h_{c}\left(\lambda, \mu^{*},(\underline{\omega}\rangle_{0}\right)$ determined by $\lambda_{c}$ is a monotonic increasing function of $\frac{\left\langle\underline{\omega}_{\omega}\right\rangle}{\omega_{0}}$ for a given $\mu^{*}$. For example, when $\mu^{*}=0.12, h_{c}$ increases from $\sim 0.035$ to 0.046 as $\frac{\left\langle\omega \omega_{0}\right\rangle}{\omega_{0}}$ increases from 0.3 to 0.9 . The premultiplier $\bar{\omega}$ also increases in the same manner as can be seen from Table I. Thus high $T_{c}^{\max }$ tends to occur in the high $\frac{\langle\omega\rangle}{\omega_{0}}$ range $\left(\mathrm{e}, \mathrm{g} . \frac{\langle\omega}{\omega_{0}} \geqslant 0,3\right)$. This is consistent with Allen's idea ${ }^{10}$ of repulsive effect of low phonon frequency on superconductivity. However, the enhancing effect due to phonon modes softening on $\lambda$ is still valid provided the softening takes place at reasonably high frequency. The softening of phonon modes is necessary to raise $\lambda$ to its critical value $\lambda_{c}^{\prime}$. When such conditions are realized, $\mathrm{T}_{\mathrm{c}}^{\max } \sim 30^{\circ} \mathrm{K}$ should be achieved. 


\section{APPENDIX}

We first derive equation (8) from equation (7). The first term on the right hand side of equation (7) is

$$
\begin{aligned}
\frac{\Delta_{\infty}}{Z_{0}} \int_{\omega_{0}}^{\infty} \frac{d \omega^{\prime}}{\omega^{\prime}} \int_{0}^{\omega_{0}} d \omega_{q} \alpha^{2} F\left(\omega_{q}\right) & \frac{2}{\omega^{\prime}+\omega_{q}} \\
& =\frac{2 \Delta_{\infty}}{Z_{0}} \int_{0}^{\omega_{0}} d \omega_{q} \alpha^{2} F\left(\omega_{q}\right) \sum_{n=1}^{\infty} \frac{1}{n} \frac{\left(-\omega_{q}\right)^{n-1}}{\omega_{0}^{n}}
\end{aligned}
$$

For $n>1$, we define $\left\langle\omega^{n}\right\rangle=\int_{0}^{\omega_{0}} \omega_{q}^{n-1} \alpha^{2} F\left(\omega_{q}\right) d \omega_{q} / \int_{0}^{\omega_{0}} \frac{d \omega}{\omega_{q}} \alpha^{2} F\left(\omega_{q}\right)$ and approximate $\left\langle\omega^{n}\right\rangle \overrightarrow{b y}\langle\omega\rangle^{n}$. This approximation will be good for $n \geq 3$ because

$$
\begin{aligned}
{\left[\frac{\int_{0}^{\omega_{0}} d \omega_{q} \alpha^{2} F\left(\omega_{q}\right)}{\int_{0}^{\omega_{0}} \frac{d \omega_{q}}{\omega_{q}} \alpha^{2} F\left(\omega_{q}\right)}\right]^{n} } & =\left[\frac{\int_{0}^{\omega_{0}} \frac{d \omega_{q}}{\omega_{q}} \alpha^{2} F\left(\omega_{q}\right) \omega_{q}}{\left.\int_{0}^{\omega_{0}} \frac{d \omega_{q}}{\omega_{q} \alpha^{2} F\left(\omega_{q}\right)}\right]^{n}}\right. \\
& \cong \frac{\int_{0}^{\omega_{0}} \frac{d \omega_{q}}{\omega_{q} \alpha^{2} F\left(\omega_{q}\right) \omega_{q}^{n}}}{\int_{0}^{\omega_{0}} \frac{d \omega_{q} \alpha^{2} F\left(\omega_{q}\right)}{\omega_{q}}}=\left\langle\omega^{n}\right\rangle
\end{aligned}
$$

in analogy to the argument used to establish equation (15). For $\mathbf{n}=2$, the right hand side is always greater than the left hand side for the strong-coupled case. Therefore, such an approximation for $n=2$ tends to give a less negative second order term in (A1) and thus a slightly more repulsive effect on $\Delta^{2}(0)$. With this approximation, the right hand side of (A 1) reduces to $\frac{\Delta_{\infty}}{Z_{0}} \lambda \ln \left(1+\frac{\langle\omega\rangle}{\omega}\right)$

The second term on the right hand side of equation (7) is 
$-28-$

$$
\begin{aligned}
& \frac{2 \Delta \infty}{Z_{0}} \int_{\omega_{0}}^{\infty} \frac{d \omega^{\prime}}{\omega^{\prime}} \int_{0}^{\omega_{0}} d \omega_{q} \alpha^{2} F\left(\omega_{q}\right) N\left(\omega_{q}\right)\left\{\frac{1}{\omega^{\prime}+\omega_{q}}+\frac{1}{\omega^{\prime}-\omega_{q}}\right\} \\
& =\frac{2 \Delta_{\infty}}{Z_{0}} \int_{0}^{2 \cdot 5 T_{c}} \frac{d \omega_{q}}{\omega_{q}} \alpha^{2} F\left(\omega_{q}\right)\left[\frac{2}{e^{\omega_{q} / T_{c}}-1}\right] \sum_{n=0}^{\infty} \frac{1}{2 n+1}\left(\frac{\omega_{q}}{\omega_{0}}\right)^{2 n+1} \\
& +\frac{2 \Delta_{\infty}}{Z_{0}} \int_{2.5 T_{c}}^{\omega_{0}} \frac{d \omega_{q}}{\omega_{q}} \alpha^{2} F\left(\omega_{q}\right) e^{-\omega_{q} / T_{c}} \ln \left|\frac{\omega_{0}+\omega_{q}}{\omega_{0}-\omega_{q}}\right| \\
& \text { where an approximation } \frac{1}{{ }_{e}^{\omega_{q} / T_{c}}} \cong e^{-w_{q} / T_{c}} \text { has been made for } \\
& \omega_{q}>2.5 \mathrm{~T}_{c} .
\end{aligned}
$$

The second term on the right hand side of (A2) can be shown to be less than $\left.\frac{2 \Delta_{\infty}}{Z_{0}}\left(\frac{T}{\omega_{0}}\right) \lambda e^{-2.5} \approx 0.02 \lambda\left(\frac{\Delta \infty}{Z_{0}}\right) \frac{\langle\omega}{\omega_{0}}\right\rangle$ for the range of $T_{c} / \omega_{0}$ considered and thus can be dropped. Also, a physical cut off has been made to remove the logarithmic singularity as $\omega_{\mathrm{q}} \rightarrow \omega_{0}$. We only retain the first power term under the summation sign in (A2) because the ratio of the second power term to the first is of order $\frac{1}{3}\left(\frac{2.5 T}{\omega_{0}}\right)^{2} \quad(\$ 0.03$ for the range of $T{ }_{c} / \omega_{0}$ considered). Grouping everything together, we rewrite equation (7) in the form

$$
\Delta_{0}^{2}=\frac{\Delta_{\infty}}{Z_{0}}\left\{\lambda \ln \left(1+\frac{\langle\omega\rangle}{\omega_{0}}\right)+4 \int_{0}^{2 \cdot 5 T_{c}} \frac{d \omega_{q}}{\omega_{0}} \alpha^{2} F\left(\omega_{q}\right)\left[\frac{1}{e^{\omega_{q} / T_{c}}-1}\right]\right\}
$$

identical to equation (8).

We proceed to evaluate the second term on the right hand side of (A3) by assuming $\alpha^{2} F \cong\left(\frac{d \alpha^{2} F}{d \omega}\right)_{0} w_{q}^{m}$ for small $\omega_{q}$ with $m$ of order unity. By substituting $x=\omega_{q} / T_{c}$ 


$$
\begin{aligned}
4 \int_{0}^{2-5 T_{c}} \frac{d \omega_{q}}{\omega_{0}} \alpha^{2} F\left(\omega_{q}\right)\left[\frac{1}{e^{\omega_{g} / T_{c}}-1}\right] \\
=4 \frac{T_{c}^{m+1}}{\omega_{0}}\left(\frac{d \alpha^{2} F}{d \omega}\right)_{0}^{2 \cdot 5} \frac{x^{m} d x}{e^{x}-1} \\
\approx \frac{4}{m}\left[\frac{1}{2}+\frac{2^{m-1}}{3}+\frac{1}{3}\left(\frac{5}{2}\right)^{m}\right]\left(\frac{d \alpha^{2} F}{d \omega}\right) \frac{T_{c}^{m+1}}{\omega_{0}}
\end{aligned}
$$

It takes the form of equation (9).

ACKNOWLEDGMENT - The author wish to thank Professor Pol Duwez for his advice and encouragement throughout this work. 


\section{REFERENCES}

Work supported by the U. S. Atomic Energy Commission. Contract No. $\mathrm{AT}(04-3)-822$.

1. J. Bardeen, L. N. Cooper, and J. R. Schrieffer, Phys. Rev. 108, $1175(1957)$.

2. B. T. Matthias, Progress in Low Temp. Phys. Vol. 2, Interscience Publishers, New York (1957), p. 138.

3. J. J. Hopfield, Phys. Rev. 186, 443 (1969).

4. D. Pines, Phys. Rev. 109, 280 (1958).

5. W. L. McMillan, Phys. Rev. 167, 331 (1967),

6. G. M. Eliashberg, Zh. Eksperim. i Teor. Fiz. 38, 966 (1960); 39, 1437 (1960). [English Transl: Soviet Phys. - JETP 11, 696 $(1960) ; 12,1000(1961)]$.

7. D. J. Scalapino, Y. Wada, and J. C. Swihart, Phys. Rev. Letters 14. $102(1965)$.

8. M. L. Cohen and P. W. Anderson; "Supérconductivity In d- and f-Band Metals". AIP Conference Proceedings No. 4. Edited by David H. Douglass.

9. B. T. Matthias, same AIP Conference Proceedings as given in reference 8 . 
10. P. B. Allen, Solid State Comm. 12, 379 (1973).

11. S. J, Poon and W. L. Johnson, to be published in Phys. Rev.

12. P. B. Allen, Proceedings of 12th Int. Conf. on Low Temp. Physics, Keigaku, Tokyo (1971). Edited by E. Kanda, p. 517.

13. L, Y. L. Shen, Phys. Rev. Letters 29, 1082 (1972).

14. T. T, Chen, I. D. Chen, J. D. Leslie, and H. J. T. Smith, Phys. Rev. Letters 22, 526 (1969).

15. R, C, Dynes, Solid State Comm. 10, 615 (1972).

16. W. L. McMillan and J. M. Rowell, Superconductivity, Marcel Dekker, New York (1969), edited by R. D. Parks. Also in Phys. Rev. Letters 19, 108 (1965).

17. J. M. Rowell, W. L. McMillan, and W. L. Feldmann, Phys. Rev. $\underline{178}, 897$ (1969).

18. W. N. Hubin and D. M. Ginsberg, Phys. Rev. 188, 716 (1969).

19, R. C. Dynes, Phys. Rev. B2, 644 (1970); Phys. Rev. B4, 3255 (1971).

20. L, Y. L. Shen, Phys. Rev. Letters 24, 1104 (1970).

21. J. C. Phillips, same AIP Proceedings as in reference 8. 